\title{
Effect of neural stem cell transplantation combined with erythropoietin injection on axon regeneration in adult rats with transected spinal cord injury
}

\author{
Y. Zhao ${ }^{1 *}$, Y. Zuo ${ }^{2 *}$, X.L. Wang ${ }^{1}$, H.J. Huo', J.M. Jiang ${ }^{3}$, H.B. Yan ${ }^{4}$ and Y.L. Xiao' ${ }^{1}$ \\ ${ }^{1}$ Department of Spinal Surgery, The Second Affiliated Hospital, \\ Inner Mongolia Medical University, Hohhot, China \\ 2Department of Clinical Laboratory, The Second Affiliated Hospital, \\ Inner Mongolia Medical University, Hohhot, China \\ ${ }^{3}$ Department of Orthopedic and Spinal Surgery, Nan-Fang Hospital, \\ Southern Medical University, Guangzhou, China \\ ${ }^{4}$ Department of Spinal Surgery, No. 3 Affiliated Hospital, Southern Medical University, \\ Guangzhou, China \\ *These authors contributed equally to this study. \\ Corresponding author: Y.L. Xiao \\ E-mail: zhaoyanneimenggu@163.com \\ Genet. Mol. Res. 14 (4): 17799-17808 (2015) \\ Received August 19, 2015 \\ Accepted October 6, 2015 \\ Published December 22, 2015 \\ DOI http://dx.doi.org/10.4238/2015.December.22.4
}

ABSTRACT. We investigated the effect of neural stem cells (NSC) and erythropoietin (EPO) on axon regeneration in adult rats with transected spinal cord injury, and provided an experimental basis for clinical treatment. Forty Wistar rats with T10-transected spinal cord injury were randomly divided into four groups of ten rats: a control group (group A), an NSC-transplant group (group B), an NSC-transplant and EPO group (group C), and an EPO group (group D). Biotinylated dextran amines (BDA) anterograde corticospinal cord neuronal tracing and Fluoro-Gold $(F G)$ retrograde tracing were carried out at the 8th week after operation 
to observe the regeneration of nerve fibers. The Basso, Beattie, and Bresnahan (BBB) locomotor score was used to evaluate restoration. 1) BDA and FG immunofluorescence staining: in group $C$, a large number of regenerated axons were observed and some penetrated the injured area. In group $\mathrm{B}$, only a small number of regenerated axons were observed and none penetrated the injured area. In group $D$, only sporadic regenerated nerve fibers were observed occasionally, while in group $A$, no axonal regeneration was observed. In group $\mathrm{C}$, a small number of cones and axons emitted yellow fluorescence, and no FG-labeled cells were observed in the other groups. 2) The BBB scores for group $C$ were higher than those for the other groups, and the differences were statistically significance $(P$ $<0.05)$. NSC transplantation combined with EPO intraperitoneal injection may benefit axon regeneration in rats with transected spinal cord injury, and accelerate the functional recovery of the hindlimb locomotor.

Key words: Neural stem cells; Erythropoietin; Axon regeneration; Cell transplantation; Spinal cord injury

\section{INTRODUCTION}

At present, spinal cord injury is the world's most common form of nerve injury and its incidence increases every year. Although in recent years there have been advances in technology and new methods for treating spinal cord injury, it still poses a major problem worldwide. In recent years, many studies have found that neural stem cells (NSC) have a unique advantage over other stem cells, and represent one of the most actively researched topics in the field. In addition, because of their unique characteristics, such as greater homology, abundance, and ease of cultivation, embryo-derived NSC are regarded as ideal stem cells for the treatment of spinal cord injury (SCI) (Cai et al., 2009; Chigr et al., 2009; Hu and Lü, 2009; Andres et al., 2011). However, the simple application of NSC to the treatment of $\mathrm{SCl}$ is not ideal. In recent years, it has been found that erythropoietin (EPO), a kind of organization protection factor (Sanchis-Gomar et al., 2013), has neurotrophic and neuroprotective effects. This poses the question of whether NSC transplantation combined with EPO can effectively promote the regeneration of injured spinal cord axons, thereby promoting functional recovery in rats with spinal cord injury. However, there have been few studies on this problem. Therefore, in this study we combined NSC and EPO for the treatment of spinal cord injury in rats. We discuss here whether the application of NSC and EPO can effectively promote axon regeneration and recovery of spinal cord function, and provide a very important theoretical basis for the clinical treatment of spinal cord injury.

\section{MATERIAL AND METHODS}

\section{Experimental animals, main reagents, and instruments}

Forty healthy adult female Wistar rats weighing $220 \pm 20 \mathrm{~g}$, including two Wistar rats that were 14 days pregnant, were provided by the animal experiment center of Inner Mongolia University (certificate No. SCXK (Mongolian) 2002-0001). The disposal of animals in the experiment met the animal ethical standards. Dulbecco's modified Eagle's medium (DMEM)/F12 media, fetal bovine 
serum (FBS), and B27 supplements were supplied by Gibco; EPO was supplied by Shenyang Junior Pharmaceutical Companies; basic fibroblast growth factor (bFGF) and epidermal growth factor (EGF) were from Sigma-Aldrich; an SABC immunohistochemical kit, nestin antibody, mice BrdU antibodies, anti-neurofilament NF-200, fluorescein isothiocyanate (FITC), trypsin, and a 25$\mathrm{cm}^{2}$ culture bottle were from Corning; the surgical instruments, fiber equipment, and an operating microscope (SXP-1C) were from Shanghai; the fluorescence microscope (TE 2000-S) was from Nikon Japan; the super clean bench (MCV-16BSU) was from Sanyo, Japan; the autoclave (HV-50) was from Hirayama, Japan; and the rat brain stereotaxic instrument was from Jiangwan, China.

\section{Primary culture and identification of NSC}

Experimental methods were conducted according to the literature (Xu et al., 2013). The pregnant rats were decapitated and we quickly removed the fetal rats by caesarean section under sterile conditions, cut the skull to obtain the brain tissue, and separated the tissue of the midbrain, hippocampus and subventricular zone using a dissecting microscope. The isolated fetal rat brain tissue was immersed in D-Hanks solution. We cut a small sample of fetal rat brain tissue $\left(1 \mathrm{~mm}^{3}\right)$ with ophthalmic scissors, digested it using an equal volume of $0.125 \%$ trypsin at $37^{\circ} \mathrm{C}$ for $10-15 \mathrm{~min}$, and repeated the procedure by mechanical pipetting, then added a double volume of $10 \%$ FBS in DMEM/F12 to terminate the digestion. The mixture was filtered through a 200-mesh stainless steel screen, centrifuged for $10 \mathrm{~min}$ at $800 \mathrm{rpm}$, and the supernatant was discarded. We then added a complete medium comprising DMEM/F12 plus B27 $(2 \mathrm{~mL} / 100 \mathrm{~mL})$, bFGF $(10 \mathrm{ng} / \mathrm{mL})$, and EGF (20 $\mathrm{ng} / \mathrm{mL}$ ). After aeration and percussion, the single cell suspension was added to flasks with living cell concentrations of $5-8 / \mathrm{mL}$. The cell suspension was cultured in a carbon dioxide incubator for 5-7 days and centrifuged; half the amount was changed once every 2-3 days. The NSC of three to four generations were made into a cell suspension, and the cell concentration was adjusted to $1 \mathrm{x}$

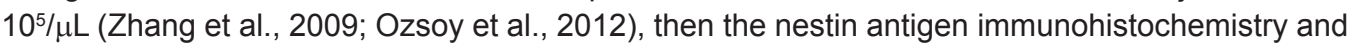
immunofluorescence were conducted.

\section{Production of the completely transected spinal cord rat model, and the experimental animal groups}

Experimental methods were conducted according to the literature (Meng et al., 2011). The rats were anesthetized with $3.5 \%$ pentobarbital $(1.3 \mathrm{~mL} / 100 \mathrm{~g}$ per rat) by intraperitoneal injection. We then exposed the T10 spinous process segments as a symbol of T8 under sterile conditions (Sanchis-Gomar et al., 2013), completely provoked the spinal cord with a blunt crochet, cut the segment of the T10 together with the dura and spinal cord tissue using a scalpel, cut 1-mm spinal segments, lifted the two ends of the transected spinal cord tissue using microscopic tweezers, and filled the spinal cord injury with a gelatin sponge.

A total of 40 adult Wistar rats were randomly divided into four groups (10 rats per group) as follows. 1) the NSC group: After modeling, the suspension of NSC was centrifuged for 5 min (1000 $\mathrm{rpm}$ ) using a tabletop centrifuge. We adjusted the cell concentration to $1 \times 10^{5} / \mu \mathrm{L}$ with DMEM, and the suspension $(10 \mu \mathrm{L})$ was injected into the local gelatin sponge. At $1 \mathrm{~mm}$ from the top and bottom of each lesion, we used a micro-syringe to inject the suspension $(0.5 \mu \mathrm{L}$ at each point) at depths of $0.25,0.5$, and $0.75 \mathrm{~mm}$. 2) the NSC + EPO group: The NSC were transplanted and EPO was injected intraperitoneally (5000 U/kg; once per day; continuous infusion for 7 days). 3) the EPO group: EPO was injected intraperitoneally (5000 U/kg; once per day; continuous infusion for 
7 days); after modeling, DMEM/F12 medium (10 $\mu \mathrm{L}$ ) was injected into the gelatin sponge. 4 ) the control group: After modeling, the DMEM/F12 medium (10 $\mu \mathrm{L}$ ) was injected into the gelatin sponge and normal saline was injected intraperitoneally.

\section{Rat hindlimb motor Basso, Beattie, and Bresnahan (BBB) score}

The BBB score (Salazar et al., 2010) was used to evaluate the hindlimb motor function of the rats. All rats were subjected to hindlimb motor function evaluation $12 \mathrm{~h}$ before surgery. At 3,7 , $14,28,42$, and 56 days following operation, two non-laboratory personnel who were familiar with the scoring carried out the randomized, double-blind score, and took an average for the final score of hindlimb motor function.

\section{Observation contents and methods}

\section{Biotinylated dextran amine (BDA) anterograde corticospinal tract tracing}

The rats were killed 21 days before the procedure and fixed in the stereotaxic apparatus. We then cut the head skin of the rats to expose the skull and Bregma point, and opened a 0.5$3.0 \mathrm{~mm}$ bone groove in the midsagittal line $2 \mathrm{~mm}$ from the Bregma point using an abrasive drill so as to reveal the dura and cut it. Using a micro-injector (the front end was provided with a glass microelectrode of less than $50 \mu \mathrm{m}$ diameter), we slowly injected $0.7 \mu \mathrm{L} 10 \%$ BDA into the Bregma point at depths of $0.5,1.75,3.0$, and $2 \mathrm{~mm}$; each rat was injected with a total of $4.2 \mu \mathrm{L}$ BDA (Wang et al., 2004; Ba et al., 2012).

\section{Fluoro-Gold (FG) retrograde tracing of the corticospinal tract}

Drawn before 10 days, the rats were anesthetized by intraperitoneal injection and fixed in the stereotaxic apparatus. We then subjected the dorsal T10 lamina to laminectomy, and cut the dura and arachnoid to clearly reveal the T12 spinal cord. At a distance of $0.5 \mathrm{~mm}$ from the sagittal midline, we injected $3 \% \mathrm{FG}(0.3 \mu \mathrm{L})$ at a rate of $0.05 \mu \mathrm{L} / \mathrm{min}$ and a depth of $1.2 \mathrm{~mm}$ using a $1-\mu \mathrm{L}$ micro-syringe; we paused for $5 \mathrm{~min}$ after each injection to prevent FG leakage, and then sutured the wounds.

\section{Draw materials}

The experimental animals survived for 8 weeks following surgery, after which $4 \%$ paraformaldehyde phosphate solution was used to fix the spinal cord tissue by cardiac perfusion. After reperfusion, we removed all laminae and calvaria bone; then, we completely removed the spinal cord and brain, and placed those tissues in a $30 \%$ sucrose solution at $4^{\circ} \mathrm{C}$.

\section{BDA fluorescence staining}

We dried the frozen sections, washed them three times with $0.01 \mathrm{M}$ KPBS for 10 min each time, and immersed them in a solution containing $0.3 \%$ Triton X-100 (0.01 M KPBS) for 30 min. We then washed them a further three times in 0.01 M KPBS and immersed the sections in 1:400 streptavidin-Сy3. We then dehydrated the sections, made them transparent, fengpian, and used a fluorescence microscope to investigate them. 


\section{FG immunofluorescence staining}

Frozen sections (30 $\mu \mathrm{m}$ thick) of coronal brain cortex tissue were obtained (one in three sections) and dehydrated, made transparent, mounted, and directly observed under a fluorescence microscope.

\section{Statistical analysis}

We used (means $\pm S D$ ) to present the results, used single-factor analysis of variance (oneway analysis of variance) to conduct statistical tests, and used the least significant difference (LSD) method to conduct pairwise comparisons. $\mathrm{P}<0.05$ was considered to be statistically significant. We used the SPSS 13.0 statistical analysis software.

\section{RESULTS}

\section{Morphological observation and immunohistochemical identification of NSC}

Single cells isolated from the hippocampus tissue of embryonic rats were round and comparably sized. Most of the cells cultured for 2-3 days died, and only a few cells entered mitosis with a significant nuclear division observed under the microscope, and dividing cells gradually formed cell clusters. After primary culture for 4-5 days, several to a dozen cells were grown in suspension. Spherical cell mass was seen in the nutrient solution; the volume of these cell clusters was small and their refraction was strong; they had no obvious bumps, were mostly brownish, had clear borders, and had edges with slight burrs, so we called them "neural spheres". After primary culture for 4-5 days, the neurospheres increased significantly; the color of the core part was deep, and the edge part was light (Figure 1A). Natural, if not timely, passage was easy. The central cells of the neurospheres were dead owing to nutrient deficiency. The neurospheres that were passaged were made into a single cell suspension and cultured for 2-3 days. The single cells suspended in the culture solution split and formed a small group of cells. When these cells were cultured for 5-6 days, we observed scattered and varied neurospheres, and these cells formed larger neurospheres after 7 days with clear boundaries and strong refraction. This shows that the hippocampal cells had the ability to self-proliferate. The neural stem cell marker (nestin protein) in the primary cultured and passaged neurospheres was expressed and those neurospheres displayed green fluorescence (Figure 1B), which indicates that those neurospheres were embryo-derived.
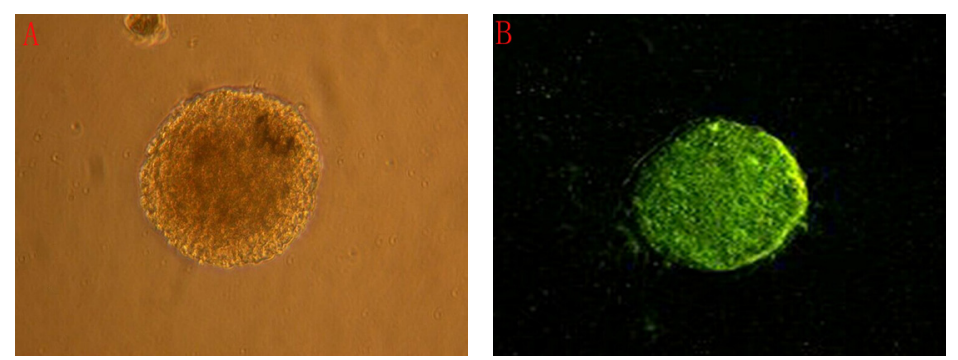

Figure 1. Nerve stem cell sphere (200X) and identifying nerve stem cell glomus by nestin immunofluorescence (200X). A. Nerve stem cell sphere (200X). B. Identifying nerve stem cell glomus by nestin immunofluorescence [fluorescein isothiocyanate (FITC) 200X]. 


\section{BDA anterograde corticospinal tract tracing}

The combined treatment group showed a large number of regenerated axons, which were labeled by BDA-Cy3 red fluorescent; some regenerated axons penetrated the damage zone and reached the distal end. While the NSC group showed a small number of regenerated axons after spinal cord injury, no regenerated axons penetrated the damage zone. The EPO group occasionally showed scattered regenerated nerve fibers, while the control group showed no axonal regeneration, and the two groups had no continuous nerve fibers that passed through the injury area (Figure 2).
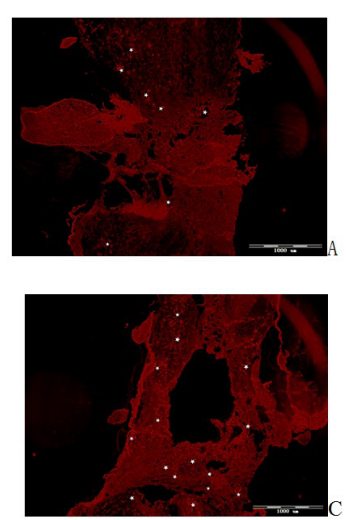
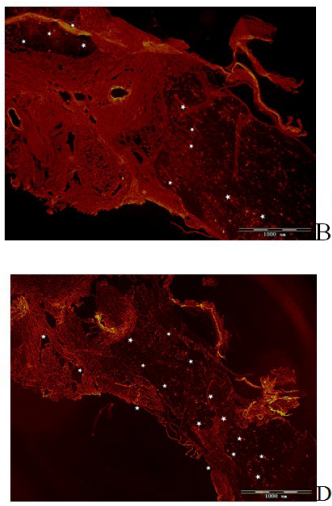

Figure 2. Corticospinal tract anterograded tracing with biotinylated dextran amines (BDA) (400X). A. Control group; B. Erythropoietin (EPO) group; C. Neural stem cell (NSC) group; D. NSC + EPO group.

\section{Corticospinal tract FG retrograde tracing}

In this study, we observed a small number of vertebral cells and axons in the combined treatment group, which were labeled by FG. However, we did not observe labeled vertebral cells and axons in other groups. Cortical vertebral cells were labeled by FG, which indirectly proved there were continuous regenerated axons penetrating the injury area in the combined treatment group, and these regenerated axons set up connections with the tail ends of nerve fibers (Figure 3 ).
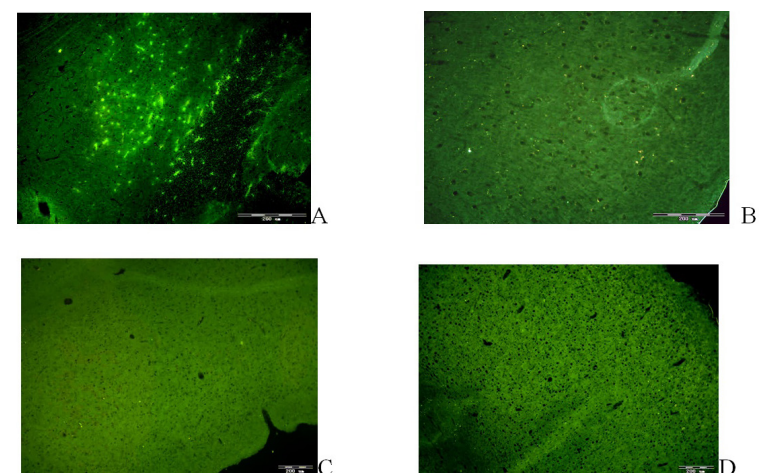

Figure 3. Corticospinal tract retrograded tracing with Fluoro-Gold (FG). A. Neural stem cell (NSC) + erythropoietin (EPO) group, pyramidal cells were labeled with $F G$, which was transported through the axon; B. NSC group; C. EPO group; D. Control group. None of the pyramidal cells was labeled with FG. 


\section{BBB scores}

The normal BBB score for a rat hindlimb is 21 points. After the spinal cord transective injury in the rats, the BBB scores of the four groups were 0 points within 3 days. With increase in time following the injury, the rat hindlimb motor function of the four groups showed different degrees of recovery, which was particularly obvious in the combined group and the NSC group, and a certain pattern was evident: after spinal cord transective injury, the motor function of the hindlimbs in the rats was lost, the function began to recover after 1 week, the recovery of the motor function was faster after 2-5 weeks, and the recovery of the motor function slowed significantly after 6-8 weeks. Comparisons between the EPO group, the NSC group, the combined group, and the control group, and a comparison between the combined group and other groups are shown in Table 1. For 1-4 weeks after the operation, the difference between the NSC group and the EPO group was not significant $(P>0.05)$; after 4 weeks, there was a statistically significant difference between the NSC group and the EPO group $(P<0.05)$, and there was a statistically significant difference between the combined group and the other groups $(P<0.05)$. At 8 weeks following the operation, we observed that the fore- and hindlimb motor function of the experimental rats was coordinated and regular, and the hind foot was weight-bearing, but still could not lift the body off the ground in the combined group. The motor coordination function was poor, and only a handful of rats had hind feet that were weight-bearing; the rest of the rats had bent toes on the hind foot, and the weight support function was poor in the NSC group. The rats' hindlimb motor function was uncoordinated and there was irregular sliding in the control and EPO groups (Table 1 and Figure 4).

Table 1. Basso, Beattie, and Bresnahan (BBB) scores of hindlimb movements following spinal cord injury (SCI) in rats (means \pm score).

\begin{tabular}{llcccc}
\hline Group & \multicolumn{5}{c}{ BBB score } \\
\cline { 2 - 6 } & $7 \mathrm{~h}$ (before) & 14 days & 28 days & 42 days & 56 days \\
\hline Control & $0.00 \pm 0.00$ & $3.06 \pm 0.36$ & $7.38 \pm 0.30$ & $7.56 \pm 0.36$ & $7.94 \pm 0.35$ \\
EPO & $0.00 \pm 0.00$ & $3.56 \pm 0.32$ & $7.39 \pm 0.34$ & $7.83 \pm 0.38$ & $8.06 \pm 0.31$ \\
NSC & $0.94 \pm 0.21^{\star}$ & $4.89 \pm 0.26^{\star}$ & $8.50 \pm 0.37^{\star}$ & $9.78 \pm 0.22^{\star}$ & $10.00 \pm 0.31^{\star}$ \\
EPO + NSC & $1.50 \pm 0.26^{\star \bullet}$ & $6.22 \pm 0.38^{\star \bullet}$ & $9.67 \pm 0.43^{\star \bullet}$ & $11.33 \pm 0.50^{*}$ & $11.61 \pm 0.46^{\star \bullet}$ \\
\hline
\end{tabular}

$\triangle$ Neural stem cells (NSC) vs control, erythropoietin (EPO), $\mathrm{P}<0.05{ }^{\star}{ }^{\bullet} \mathrm{EPO}+\mathrm{NSC}$ vs NSC vs control and EPO group, $P<0.05$. Repeated measurements follow the least significant difference (LSD) test.

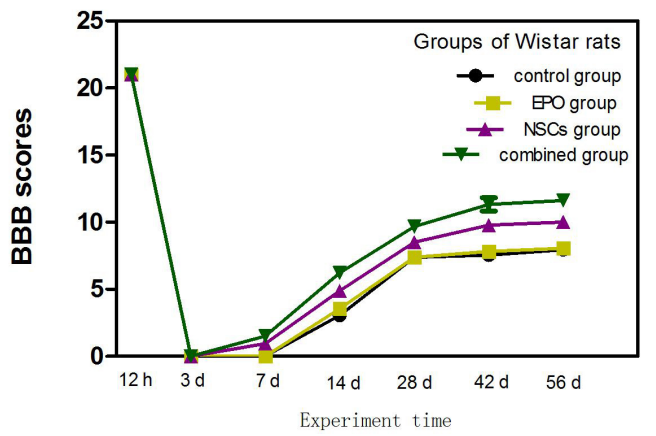

Figure 4. Basso, Beattie, and Bresnahan (BBB) score for hindlimb movements following spinal cord injury (SCl) in adult rats. Annotation: $12 \mathrm{~h}$ before the injury, 7 days (d) after the injury, 14 days after the injury, 28 days after the injury, 42 days after the injury, 56 days after the injury. 


\section{DISCUSSION}

Regeneration and repair following spinal cord injury are difficult. Research has revealed that the possible mechanism by which NSC function in the treatment of spinal cord injury is as follows.

1) When NSC are transplanted into the host body, the transplanted cells migrate toward the site of the $\mathrm{SCl}$, and under the influence of chemokines, differentiate into the various cells of the nervous system, replace damaged nerve cells, and rebuild synaptic connections, which can promote the recovery of neurological function. Nakamura et al (2005) found that the transplanted NSC can survive, differentiate, and migrate in the host body, and can effectively promote the recovery of motor function in the limbs.

2) The transplanted NSC have neurotrophic effects, and can differentiate into neurons and glial cells in the area of the SCl. These nerve cells can secrete a variety of neurotrophic factors, which can improve the local internal environment of the $\mathrm{SCl}$, promote the regeneration of axons, repair damaged nerve cells, and activate the sequential expression of regeneration-related genes. Furthermore, the transplanted cells can also produce a variety of extracellular matrices, filling the cavity left by the $\mathrm{SCl}$ and providing support for the regeneration of axons.

3) The transplanted NSC can differentiate into oligodendrocytes, which can promote the regeneration of nerve fibers and myelinize the remaining demyelinated nerve fibers (Andres et al., 2011; Garbossa et al., 2012). If the problems mentioned above are solved, it is possible to overcome the challenges of SCl. Currently, the means of assessing regeneration and repair in $\mathrm{SCl}$ is to observe whether there is regeneration of axons. The main frame of neurons and axons comprises neurofilament protein (NF). However, after SCI, NF also gradually collapses and is reduced with the degeneration and necrosis of the axons, which results in the formation of an injured spinal cord cavity or scar tissue. Therefore, NF expression is closely linked to axon regeneration.

NSC are stem cells with multiple differentiation potential, and can not only proliferate, but also self-renew and differentiate into astrocytes, neurons, and oligodendrocytes. Therefore, they are an ideal seed cell for transplantation. In this study, the NSC extracted from the fetal rat hippocampus were cultured in vitro, which significantly increases the number, activity, and purity of the primary NSC. We also observed that these cells have favorable cloning ability, and can be stably passaged. However, the proportion of NSC in vivo that naturally differentiate into neurons is only $1 \%$; the proportion of glial cells and oligodendrocytes is 31.2 and $50.3 \%$, respectively (Parr et al., 2007). The results of this study showed that the proportion of NSC in vivo that naturally differentiated into neurons was less than $5 \%$, which seriously affected the ability of the NSC transplantation to effect repair in the SCI. Therefore, inducing NSC differentiation into neurons has become the focus of current research. Watt and Hermanson et al. (2002) used a variety of neural growth factors or drugs to induce the differentiation of NSC in vitro, but the proportion of neurons was reported inconsistently. In the past, it was believed that EPO was an endocrine hormone that only acted on hematopoietic cells. However, further research has shown that EPO is a kind of new tissue protection factor that has neurotrophic and neuroprotective effects. Yuan et al. (2011) observed the effects of erythropoietin on the differentiation of embryonic cerebral cortex neural stem cells of rats cultured in vitro; the results showed EPO can promote the differentiation of NSC into neurons. Therefore, in this study we combined NSC and EPO for the treatment of $\mathrm{SCl}$ in rats. The hope was that the effect of EPO on the proliferation and differentiation of neural stem cell could effectively promote axonal regeneration and functional restoration, thereby providing a theoretical basis for the clinical treatment of $\mathrm{SCl}$.

In this study, we adopted the transected spinal cord method to produce a model of rat SCl. 
Although it cannot simulate clinical $\mathrm{SCl}$, the method can make the injury in each group uniform, so as to make the results more reliable. After the modeling, if no interventions are applied following the operation, the BBB scores were generally under 10 points, mostly eight (Meng et al., 2011). These research data showed that the hindlimb motor function in each group did not recover significantly within 7 days of $\mathrm{SCl}$. The hindlimb motor function in each group recovered to different degrees within 7-56 days of SCl. The hindlimb function recovery in the combined group was significantly better than in the other groups, but the difference between the control group and the EPO group was not statistically significant. The hindlimb function recovery in each group was faster within 7-28 days of $\mathrm{SCl}$, but we observed that the BBB score of the combined group reached more than 10 points 28 days after the operation, which has a certain significance for the recovery of spinal cord function. It has been found that cortical motor neurons regulate the growth and orientation of the nerve in the corticospinal tract, and the metabolism and physiological functions of nerves also depend on nutrients transported by the axoplasm from neurons. It was observed in the experiment that there was a significant difference between the BBB score and NF regeneration in the NSC and control groups. Particularly in the combined and NSC groups, a lot of nerve fiber regeneration of messy, disorderly appearance was visible in the cross-sectional area, and a lot of continuous nerve fibers penetrated the cross-sectional area in the combined group. Unfortunately, although we attempted to treat $\mathrm{SCl}$ using NSC combined with EPO, and continuous nerve fibers did penetrate the cross-sectional area, hind limb function in the rats showed only partial improvement and did not attain the expected outcome.

Overall, the results of this study showed that neural stem cells combined with erythropoietin can promote axon regeneration, and the regeneration of axons reached the damaged zone and passed through the caudal of the injured area. BDA anterograde corticospinal tract tracing showed a large number of regenerated axons, which were labeled by BDA-Cy3 red fluorescence; some regenerated axons penetrated the damage zone and reached the distal end. Corticospinal tract FG retrograde tracing showed cortical vertebral cells labeled with FG, which indirectly proved that continuous regenerated axons were penetrating the injury area in the combined treatment group, and these regenerated axons set up connections with the tail ends of nerve fibers. Therefore, NSC transplantation combined with EPO is a significant and promising treatment strategy for axonal regeneration and functional recovery in rats with spinal cord injury.

\section{Conflicts of interest}

The authors declare no conflict of interest.

\section{ACKNOWLEDGMENTS}

Research supported by grants from the Inner Mongolia Natural Science Foundation (\#2011ms1139).

\section{REFERENCES}

Andres RH, Horie N, Slikker W, Keren-Gill H, et al. (2011). Human neural stem cells enhance structural plasticity and axonal transport in the ischaemic brain. Brain 134: 1777-1789.

Ba YC, Tang JZ, Fan Y, et al. (2012). Demonstration empirical study of the corticospinal tracts by biotinylated dextran amine tracer. J. Clin. Rehabilit. Tissue Eng. Res. 16: 4447-4452.

Cai PQ, Sun GY, Cai PS, Martin Oudega, et al. (2009). Survival of transplanted neurotrophin-3 expressing human neural stem cells and motor function in a rat model of spinal cord injury. Neural Regen. Res. 4: 485-491. 
Chigr F, Rachidi F, Segura S, Mahaut S, et al. (2009). Neurogenesis inhibition in the dorsal vagal complex by chronic immobilization stress in the adult rat. Neuroscience 158: 524-536.

Garbossa D, Boido M, Fontanella M, Fronda C, et al. (2012). Recent therapeutic strategies for spinal cord injury treatment: possible role of stem cells. Neurosurg. Rev. 35: 293-311.

Hermanson O, Jepsen K and Rosenfeld MG (2002). N-CoR controls differentiation of neural stem cells into astrocytes. Nature 419: 934-939.

Hu M and Lü G (2009). Neural stem cell transplantation in the treatment of spinal cord injury. J. Clin. Rehabilit. Tissue Eng. Res. 13: 3751-3754.

Meng BL, Ba YC, Song SN, et al. (2011). Establishment of spinal cord transection injury models in rats. J. Clin. Rehabilit. Tissue Eng. Res. 15: 1215-1218.

Nakamura M, Toyama Y and Okano H (2005). Transplantation of neural stem cells for spinal cord injury. Rinsho Shinkeigaku 45: 874-876.

Ozsoy O, Ozsoy U, Stein G, Semler O, et al. (2012). Functional deficits and morphological changes in the neurogenic bladder match the severity of spinal cord compression. Restor. Neurol. Neurosci. 30: 363-381.

Parr AM, Kulbatski I and Tator $\mathrm{CH}$ (2007). Transplantation of adult rat spinal cord stem/progenitor cells for spinal cord injury. J. Neurotrauma 24: 835-845.

Salazar DL, Uchida N, Hamers FP, Cummings BJ, et al. (2010). Human neural stem cells differentiate and promote locomotor recovery in an early chronic spinal cord injury NOD-scid mouse model. PLoS One 5: e12272.

Sanchis-Gomar F, Perez-Quilis C and Lippi G (2013). Erythropoietin receptor (EpoR) agonism is used to treat a wide range of disease. Mol. Med. 19: 62-64.

Wang XX, Zha WG and Yi LH (2004). BDA anterograde corticospinal cord neuronal tracing in the rat models of spinal cord injury. Chin. J. Neurosurg. 20: 51-54.

Xu FC, Zou LL, Mei XM and Guo Y (2013). Culture of neural stem cells and the influencing factors. J.Clin. Rehabilit. Tissue Eng. Res. 17: 1835-1840.

Yuan LL, Du HM, Guan YJ, Kong You-hua, et al. (2011). Differentiation of rat embryonic cerebral cortex neural stem cells cultured by erythropoietin in vitro. J. Clin. Rehabilit. Tissue Eng. Res. 15: 9174-9177.

Zhang DJ, Liu Y, Fu CF, et al. (2009). Separation, cultivation and differentiation of neural stem cells from newborn rat spinal cords. J. Jilin Univ. (Medicine Edition) 35: 230-233. 